\title{
VIDA ÚTIL DE PÊSSEGOS 'AURORA 2’ ARMAZENADOS SOB ATMOSFERA MODIFICADA E REFRIGERAÇÃO'
}

\author{
ELISÂNGELA ELENA NUNES², BRÍGIDA MONTEIRO VILAS BOAS ${ }^{3}$, GENY LOPES DE CARVALHO ${ }^{4}$, \\ HELOÍSA HELENA DE SIQUEIRA², LUIZ CARLOS DE OLIVEIRA LIMA ${ }^{5}$
}

\begin{abstract}
RESUMO - O objetivo deste trabalho foi avaliar a eficiência da película de fécula de mandioca 3\% e de sacos plásticos de polietileno de baixa densidade na conservação da qualidade pós-colheita de pêssegos 'Aurora 2 ' armazenados sob refrigeração ( $9 \pm 1{ }^{\circ} \mathrm{C}$ e $90 \pm 5 \%$ UR) por 10 dias. $\mathrm{O}$ experimento foi realizado no DCA/Ufla, em Lavras-MG. A fécula 3\% induziu maior perda de massa, comprometendo a qualidade final do fruto e tendo vida útil de apenas 6 dias. O uso do polietileno reduziu a perda de massa e manteve a firmeza, diminuindo a solubilização das pectinas.
\end{abstract}

Termos para indexação: Prunus persica (L.) Batsch, pós-colheita, fécula de mandioca, filme de polietileno

\section{SHELF-LIFE OF PEACHES 'AURORA 2' STORED IN MODIFIED ATMOSPHERE AND REFRIGERATION}

\begin{abstract}
The goal of the present work was to evaluate the use of starch film of cassava and polyethylene package in postharvest quality maintenance of peaches 'Aurora 2 ' stored under refrigeration $\left(9 \pm 1{ }^{\circ} \mathrm{Ce} 90 \pm 5 \% \mathrm{RH}\right)$ for 10 days. This experiment was carried out in DCA/Ufla, Lavras, MG. The film at the concentration of 3\% showed higher mass loss and compromising the final quality, with shelf-life only for 6 days. The fruits packed with polyethylene bags presented reduction of mass loss and firmness maintenance due to pectin solubilization decrease.
\end{abstract}

Index terms: Prunus persica (L.) Batsch, postharvest, starch film of cassava, polyethylene

\section{INTRODUÇÃO}

O pessegueiro (Prunus persica (L). Batsch) pertence à família Rosaceae e é nativo da China (Medeiros \& Raseira, 1998). É um fruto muito perecível, necessitando de técnicas que ampliem sua vida útil, tais como o uso de refrigeração e atmosfera modificada (AM).

As barreiras artificiais usadas em AM podem ser genericamente de dois tipos: revestimentos e filmes plásticos (Smith et al., 1987), permitindo que a concentração de $\mathrm{CO}_{2}$ proveniente do próprio produto aumente, e a concentração de $\mathrm{O}_{2}$ diminua ao redor do mesmo, à medida que é utilizado pelo processo respiratório. Neste tipo de armazenamento, as concentrações de $\mathrm{O}_{2}$ e $\mathrm{CO}_{2}$ não são controladas e variam com a temperatura, tipo de filme e taxa respiratória do produto (Chitarra \& Prado, 2000).

A mandioca é um produto nacional e excelente fonte de amido. O amido extraído da mandioca apresenta boas características para formação de películas que, além de serem comestíveis, são de baixo custo quando comparadas às ceras comerciais. A obtenção de película de fécula de mandioca baseia-se no princípio da gelatinização do amido que ocorre acima de $70^{\circ} \mathrm{C}$ com excesso de água. Após resfriado, forma películas transparentes e resistentes, devido a suas propriedades de retrogradação. Películas de fécula de mandioca representam, assim, alternativa potencial na conservação pós-colheita de frutas e hortaliças (Oliveira, 2000).

O objetivo deste trabalho foi prolongar a vida pós-colheita de pêssegos 'Aurora 2', submetidos à atmosfera modificada, pelo uso de película de fécula de mandioca e sacos de polietileno de baixa densidade, armazenados sob refrigeração por 10 dias.

\section{MATERIALEMÉTODOS}

Os pêssegos da cultivar Aurora 2 foram adquiridos de um pomar comercial no município de Lavras-MG. Os frutos foram colhidos pela manhã e selecionados quanto à ausência de defeitos e estádio de maturação (verde-maturo). Em seguida, foram transportados para 0 Laboratório de Bioquímica de Frutos (pós-colheita) do Departamento de Ciência dos Alimentos (DCA) da Universidade Federal de Lavras (Ufla), onde foram novamente selecionados para a uniformização do grau de maturação. Os frutos foram sanificados em solução de hipoclorito de sódio 200ppm, por 15 minutos, e secos à temperatura ambiente.

Os pêssegos foram distribuídos aleatoriamente em 3 grupos: 1Controle: os frutos colocados em bandejas de isopor; 2- Fécula de mandioca 3\%: os frutos foram imersos por 1 minuto, deixados secar e colocados em bandejas de isopor; 3- Sacos de polietileno de baixa densidade (60 $\mathrm{m}$ de espessura): os frutos foram colocados em bandejas de isopor e estas acondicionadas dentro de sacos de polietileno fechados.

O experimento foi conduzido no delineamento inteiramente casualizado, com 3 repetições, sendo os tratamentos arranjados no esquema fatorial $3 \times 6$, sendo 3 níveis do fator atmosfera modificada (controle, fécula $3 \%$ e polietileno) e 6 níveis do fator tempo de armazenamento $(0 ; 2 ; 4 ; 6 ; 8$ e 10 dias). A parcela experimental foi constituída por 7 frutos.

Para o preparo da fécula de mandioca $3 \%$, foram pesados $60 \mathrm{~g}$ de fécula de mandioca, completado o volume para $2 \mathrm{~L}$ com água destilada, aquecida a mistura a $70^{\circ} \mathrm{C}$ para gelatinização (15 minutos) e deixado resfriar até a temperatura ambiente.

As bandejas foram armazenadas por 10 dias sob refrigeração $(9$ $\pm 1^{\circ} \mathrm{C}$ e $90 \pm 5 \%$ UR). As análises foram realizadas a cada 2 dias, determinando: a) perda de massa (\%), calculada pela diferença entre a massa inicial e a obtida em cada tempo de armazenamento, utilizando balança semi-analítica; b) acidez total titulável (\% ácido málico), por titulometria (Instituto Adolf Lutz, 1985); c) pH, com o auxílio de pHmetro (AOAC, 1992); d) sólidos solúveis totais ( $\left.{ }^{\mathrm{B} B r i x}\right)$, com refratômetro digital ATAGO PR-100 (AOAC, 1992); e) açúcares solúveis totais (\% glicose), pelo método de antrona (Dische, 1962); f) firmeza (N), utilizando penetrômetro Mc Cormick FT327 com ponteira de $11 \mathrm{~mm}$, e g) pectinas total e solúvel (mg de ácido galacturônico por $100 \mathrm{~g}$ polpa), extraídas segundo McCready \& McComb (1952) e os teores determinados colorimetricamente segundo Bitter \& Muir (1962).

Visto que os frutos tratados com fécula $3 \%$ só alcançaram 8 dias de armazenamento, procedeu-se a análise estatística, considerando o experimento mencionado anteriormente como um fatorial desbalanceado. As análises estatísticas foram realizadas no programa SAS versão 6.12, 1998. Os resultados significativos apresentados pela análise de variância foram submetidos ao teste de Tukey, fixando o nível

\footnotetext{
${ }^{1}$ (Trabalho 064/2004). Recebido: 03/05/2004. Aceito para publicação: 08/12/2004

${ }^{2}$ Farmacêutica-Bioquímica, Msc, doutoranda em Ciência dos Alimentos, Departamento de Ciência dos Alimentos - DCA, Universidade Federal de Lavras - UFLA, Lavras, MG elinunes@ufla.br

${ }^{3}$ Engenheira Agrônoma, Msc, doutoranda em Ciência dos Alimentos, DCA-UFLA bmvboas@hotmail.com.

${ }^{4}$ Bióloga, mestranda em Ciência dos alimentos, DCA-UFLA.

${ }^{5}$ Farmacêutico-Bioquímico, Prof. Dr. em Ciência dos Alimentos, DCA-UFLA lcolima@ufla.br.
}

Rev. Bras. Frutic., Jaboticabal - SP, v. 26, n. 3, p. 438-440, Dezembro 2004 
de significância em 5\%, para os tratamentos qualitativos (controle, fécula $3 \%$ e polietileno), e para os tratamentos quantitativos, foi realizado um estudo de regressão.

\section{RESULTADOS E DISCUSSÃO}

A interação entre atmosfera modificada e o tempo de armazenamento foi significativa para a variável perda de massa $(\mathrm{p} \leq 0,05)$. O polietileno determinou menor perda de massa em relação ao controle e fécula $3 \%$, ao longo do período de armazenamento (Tabela 1), atingindo esta perda mínima, no décimo dia de armazenamento, $0,47 \%$. Tais resultados concordam com os obtidos por Kluge et al. (1999) que, testando o uso de bandejas plásticas cobertas com filme de PVC esticável, ensacadas com polietileno de alta densidade $(20 \mu \mathrm{m})$ e de baixa densidade $(70 \mu \mathrm{m})$ na conservação pós-colheita de pêssegos 'Flordaprince' refrigerados, observaram, aos 14 dias de armazenamento, que as embalagens de polietileno foram mais eficientes em reduzir a perda de massa dos frutos $(0,54 \%)$ por promoverem maior umidade relativa, reduzindo o déficit de pressão de vapor e, conseqüentemente, a transpiração.

Os frutos tratados com fécula $3 \%$ apresentaram maior perda de massa $(19,72 \%)$, sendo que, no oitavo dia, estavam impróprios para comercialização, com aspecto enrugado. Segundo Oliveira (2000), a fécula de mandioca $2 \%$ não reduziu a perda de massa fresca de pêssegos 'Biuti' armazenados por 35 dias sob refrigeração $\left(4 \pm 2^{\circ} \mathrm{Ce} 90 \pm \mathbf{5 \%}\right.$ UR), tendo sido da ordem de $13,14 \%$ após 2 semanas de armazenamento.

A acidez total titulável (ATT) não foi influenciada pelos fatores atmosfera modificada e tempo de armazenamento, nem pela interação entre ambos $(\mathrm{p}>0,05)$, apresentando os frutos média de $0,23 \%$ de ácido málico.

Houve interação significativa entre os fatores atmosfera modificada e tempo de armazenamento para a variável $\mathrm{pH}(\mathrm{p} \leq 0,05)$, sendo esta diferença detectada a partir do quarto dia de armazenamento, com os frutos-controle apresentando os menores valores de $\mathrm{pH}$ e os demais tratamentos praticamente não diferindo entre si (Tabela 2).

TABELA 1 - Valores médios de perda de massa (\%) de pêssegos 'Aurora $2^{\prime}$ armazenados sob refrigeração $\left(9 \pm 1{ }^{\circ} \mathrm{Ce} 90 \pm 5 \%\right.$ UR) por 10 dias.

\begin{tabular}{lcccccc}
\hline & \multicolumn{7}{c}{ Tempo de armazenamento (dias) } \\
\cline { 2 - 7 } & 0 & 2 & 4 & 6 & 8 & 10 \\
\hline Controle & 0 & $3,36 \mathrm{~b}$ & $5,68 \mathrm{~b}$ & $7,85 \mathrm{~b}$ & $9,85 \mathrm{~b}$ & $11,48 \mathrm{a}$ \\
Fécula 3\% & 0 & $7,79 \mathrm{a}$ & $12,51 \mathrm{a}$ & $16,26 \mathrm{a}$ & $19,72 \mathrm{a}$ & - \\
Polietileno & 0 & $0,05 \mathrm{c}$ & $0,24 \mathrm{c}$ & $0,33 \mathrm{c}$ & $0,39 \mathrm{c}$ & $0,47 \mathrm{~b}$ \\
\hline
\end{tabular}

Médias seguidas de mesma letra na coluna não diferem entre si, pelo teste de Tukey, a $5 \%$. $\mathrm{CV}=6,8 \%$.

TABELA 2 - Valores médios de pH de pêssegos 'Aurora 2' armazenados sob refrigeração $\left(9^{ \pm} 1^{\circ} \mathrm{C}\right.$ e $90 \pm 5 \%$ UR) por 10 dias.

\begin{tabular}{lcccccc}
\hline & \multicolumn{6}{c}{ Tempo de armazenamento (dias) } \\
\cline { 2 - 7 } & 0 & 2 & 4 & 6 & 8 & 10 \\
\hline Controle & $4,57 \mathrm{a}$ & $4,44 \mathrm{a}$ & $4,50 \mathrm{~b}$ & $4,52 \mathrm{c}$ & $4,56 \mathrm{~b}$ & $4,58 \mathrm{~b}$ \\
Fécula 3\% & $4,57 \mathrm{a}$ & $4,49 \mathrm{a}$ & $4,65 \mathrm{a}$ & $4,74 \mathrm{a}$ & $4,71 \mathrm{a}$ & - \\
Polietileno & $4,57 \mathrm{a}$ & $4,50 \mathrm{a}$ & $4,63 \mathrm{a}$ & $4,65 \mathrm{~b}$ & $4,67 \mathrm{a}$ & $4,70 \mathrm{a}$ \\
\hline
\end{tabular}

Médias seguidas de mesma letra na coluna não diferem entre si, pelo teste de Tukey, a $5 \%$. CV $=1,06 \%$

A variável sólidos solúveis totais (SST) não foi afetada pelos tratamentos $(\mathrm{p}>0,05)$, cuja média encontrada foi de $12,4^{\circ}$ Brix. Segundo Oliveira \& Cereda (2003), os teores de SST de pêssegos 'Biuti' não variaram significativamente durante o período de armazenamento (12 dias) sob condições ambiente, apresentando valores na faixa de 7,9 a $10,1^{\circ}$ Brix, submetidos a diferentes tratamentos. Kluge et al. (1999) observaram que o teor de SST em pêssegos 'Flordaprince' refrigerados e acondicionados em embalagens plásticas não diferiu entre si, diferindo apenas dos frutos-controle.
A variável açúcares solúveis totais (AST) foi afetada significativamente somente pelo fator tempo de armazenamento $(p \leq 0,01)$. Houve aumento nos valores de AST até o sexto dia (Figura 1) devido, provavelmente, à perda de massa. Após este período, ocorreu pequena redução, ocasionada possivelmente pelo uso de açúcares como substratos respiratórios.

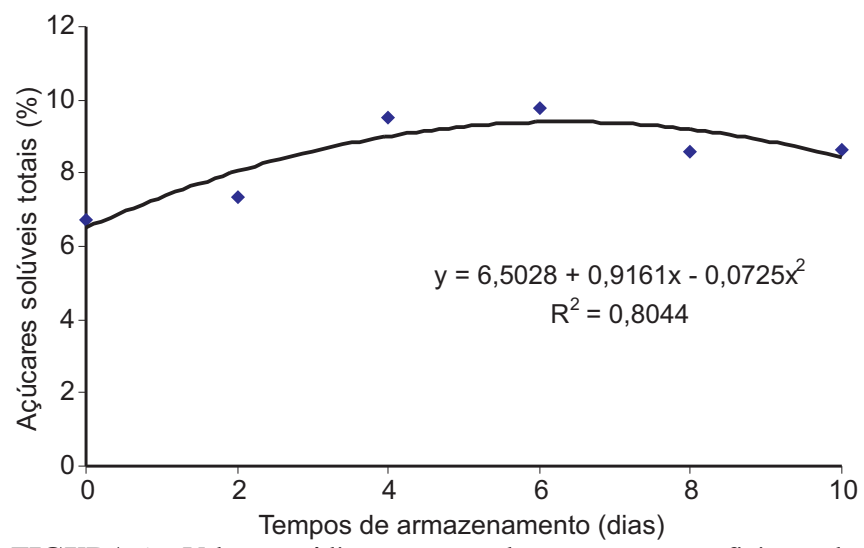

FIGURA 1 - Valores médios, equação de regressão e coeficiente de determinação de açúcares solúveis totais de pêssegos 'Aurora $2^{\prime}$ armazenados sob refrigeração $\left(9 \pm 1^{\circ} \mathrm{C}\right.$ e $90 \pm$ $5 \%$ ) por 10 dias. $\mathrm{CV}=12,61 \%$.

Houve interação significativa entre os fatores atmosfera modificada e tempo de armazenamento para a variável firmeza $(\mathrm{p} \leq 0,05)$. A firmeza inicial dos pêssegos foi de $63,62 \mathrm{~N}$ (Tabela 3). Os frutos-controle apresentaram firmeza inferior à dos demais tratamentos durante os 10 dias de armazenamento. Os frutos tratados com fécula $3 \%$ apresentaram maior valor de firmeza comparados aos embalados em polietileno, no sexto dia de armazenamento, provavelmente por terem apresentado maior perda de massa, tornando-os mais resistentes à força de penetração. Oliveira (2000) não verificou diferença significativa entre os pêssegos 'Biuti' tratados com 'fruit wax', fécula de mandioca $2 \%$ e microemulsão, armazenados por 35 dias sob refrigeração. Segundo Kluge et al. (1999), pêssegos 'Flordaprince', armazenados por 14 dias sob refrigeração e submetidos a diferentes embalagens plásticas, apresentaram maiores valores de firmeza $(47,92 \mathrm{~N})$ quando embalados em polietileno de baixa densidade $(70 \mu \mathrm{m})$ em relação às revestidas por PVC esticável e polietileno de alta densidade $(20 \mu \mathrm{m})$. O uso de polietileno de baixa densidade modificou a atmosfera no interior da embalagem, retardando o amadurecimento, o que resultou na maior manutenção da firmeza em relação ao controle.

TABELA 3 - Valores médios de firmeza $(\mathrm{N})$ de pêssegos 'Aurora 2' armazenados sob refrigeração $\left(9 \pm 1^{\circ} \mathrm{C}\right.$ e $90 \pm 5 \%$ UR $)$ por 10 dias.

\begin{tabular}{lcccccc}
\hline & \multicolumn{6}{c}{ Tempo de armazenamento (dias) } \\
\cline { 2 - 7 } & 0 & 2 & 4 & 6 & 8 & 10 \\
\hline Controle & 63,62 a & 57,06 b & 43,24 b & 28,41 c & 22,24 b & 21,29 b \\
Fécula 3\% & 63,62 a & 62,55 a & 51,05 a & 47,64 a & 38,49 a & - \\
Polietileno & 63,62 a & 62,45 a & 48,81 a & $36,99 \mathrm{~b}$ & 33,64 a & 30,01 a \\
\hline
\end{tabular}

Médias seguidas de mesma letra na coluna não diferem entre si, pelo teste de Tukey, a $5 \%$. CV $=5,37 \%$.

A variável pectina total foi afetada significativamente apenas pelo fator tempo de armazenamento $(\mathrm{p} \leq 0,01)$, sendo observado pequeno aumento nos seus teores durante o armazenamento (Figura 2). Houve interação significativa entre os fatores atmosfera modificada e tempo de armazenamento para a variável pectina solúvel $(\mathrm{p} \leq 0,01)$. A partir do sexto dia de armazenamento (Tabela 4), os frutos-controle apresentaram os maiores teores de pectina solúvel, possivelmente devido à atuação das enzimas da parede celular, provocando maior solubilização das 
TABELA 4 - Valores médios de pectina solúvel (mg ácido galacturônico. $\left.100 \mathrm{~g}^{-1}\right)$ de pêssegos 'Aurora 2 ' armazenados sob refrigeração $\left(9 \pm 1{ }^{\circ} \mathrm{Ce} 90 \pm\right.$ $5 \%$ UR) por 10 dias.

\begin{tabular}{lccccc}
\hline & \multicolumn{4}{c}{ Tempo de Armazenamento (dias) } \\
\cline { 2 - 5 } & 0 & 2 & 4 & 6 & 10 \\
\hline Controle & $33,4 \mathrm{a}$ & $36,69 \mathrm{a}$ & $56,55 \mathrm{a}$ & $114,48 \mathrm{a}$ & $190,79 \mathrm{a}$ \\
Fécula 3\% & $33,4 \mathrm{a}$ & $37,31 \mathrm{a}$ & $51,99 \mathrm{a}$ & $67,15 \mathrm{~b}$ & $96,31 \mathrm{~b}$ \\
Polietileno & $33,4 \mathrm{a}$ & $32,97 \mathrm{a}$ & $45,76 \mathrm{a}$ & $66,31 \mathrm{~b}$ & $95,65 \mathrm{~b}$ \\
\hline
\end{tabular}

Médias seguidas de mesma letra na coluna não diferem entre si, pelo teste de Tukey, a 5\%.CV=12,45\%.

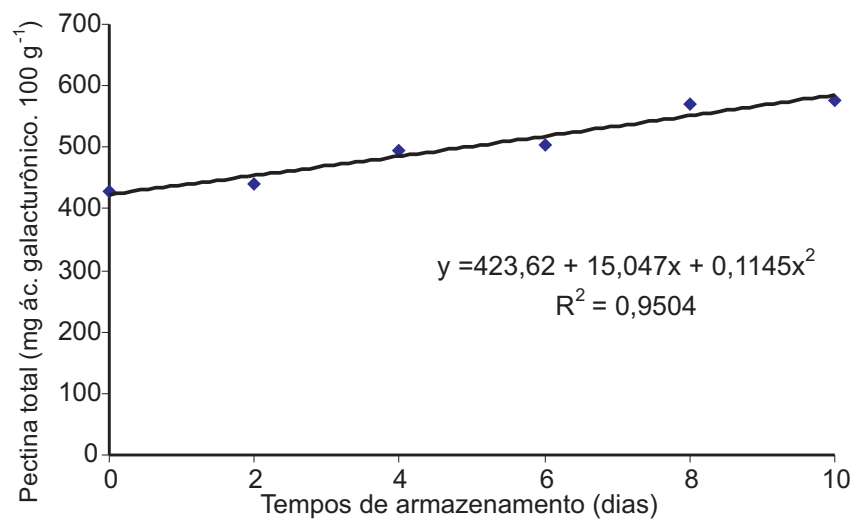

FIGURA 2 - Valores médios, equação de regressão e coeficiente de determinação de pectina total de pêssegos 'Aurora 2' armazenados sob refrigeração $\left(9 \pm 1^{\circ} \mathrm{C}\right.$ e $\left.90 \pm 5 \%\right)$ por 10 dias. $\mathrm{CV}=11,11 \%$.

substâncias pécticas e, conseqüentemente, menores valores de firmeza (Tabela 3).

\section{CONCLUSÕES}

1) A fécula 3\% não é eficiente em prolongar a vida útil de pêssegos 'Aurora 2' armazenados sob refrigeração, permitindo a perda excessiva de massa, tornando os frutos impróprios para comercialização a partir do oitavo dia de armazenamento.

2) O polietileno mostra-se efetivo na manutenção da qualidade e extensão pós-colheita por 10 dias, determinando mínimas perdas de massa durante o período de armazenamento, maior firmeza e menor solubilização das pectinas.

\section{REFERÊNCIAS}

ASSOCIATION OF OFFICIALANALYTICAL CHEMISTRY. Official methods of analysis of the Association of Official Analytical
Chemistry. 12.ed. Washington, 1992. 1.015p.

BITTER, T.; MUIR, H. M. A modified uronic acid carbazole reaction. Analytical Biochemistry, New York, v. 34, p. 330-334, 1962.

CHITARRA, A. B.; PRADO, M. E. T. Utilização de atmosfera modificada e controlada em frutos e hortaliças. Lavras: UFLA/ FAEPE, 2000. 66p.

DISCHE, Z. General color reactions. In: WHISTLER, R. L.; WOLFRAM, M. L. Carbohydrate chemistry. New York: Academic Press, 1962. p. 477-512.

INSTITUTO ADOLFO LUTZ. Normas analíticas, métodos químicos e físicos para análise de alimentos. 3.ed. São Paulo: Instituto Adolfo Lutz, 1985. v. 1, 533p.

KLUGE, R. A.; SCARPARE FILHO, J. A.; JACOMINO, A. P.; MARQUES, C. Embalagens plásticas para pêssegos 'Flordaprince' refrigerados. Scientia Agrícola, Piracicaba, v. 56, n. 4, p. 843-850, out/dez. 1999.

McCREADY, R. M.; McCOMB, E. A. Extraction and determination of total pectic materials in fruit. Analytical Chemistry, Washington, v. 24, n. 12, p. 1586-1588, Dec. 1952.

MEDEIROS, C. A. B.; RASEIRA, M. C. B. A cultura do pessegueiro Brasília: Embrapa-SPI; Pelotas: Embrapa-CPACT, 1998. 350p.

OLIVEIRA, M. A. de. Comportamento pós-colheita de pêssegos (Prunus persica L. Bastsch) revestidos com filmes à base de amido como alternativa à cera comercial, 2000. 98f. Tese (Doutorado em Agronomia) - Faculdade de Ciências Agronômicas, Universidade Estadual Paulista, Botucatu, 2000.

OLIVEIRA, M. A. de; CEREDA, M. P. Pós-colheita de pêssegos (Prunus persica (L.) Batsch) revestidos com filmes à base de amido como alternativa à cera comercial. Ciência e Tecnologia de Alimentos, Campinas, v.23 (Supl), p. 28-33, dez. 2003.

SAS INSTITUTE. SAS user's guide: statistic - version 6.12. ed. Cary, SAS INSTITUTE, 1998. 846p.

SMITH, S.; GEESON, J.; STOW, J. Production of modified atmospheres in deciduous fruits by the use of films and coatings. HortScience, Alexandria, v. 22, n. 5, p. 772-776, 1987. 